\title{
BIOLOGICAL EFFECTS OF RADIATION
}

$A^{\mathrm{T}}$ the Second United Nations International Conference on the Peaceful Uses of Atomic Energy only two of the sessions on isotopes and biology were devoted to what might strictly be called radiobiology. Health and safety in the atomic energy industry and advances in the use of isotopes (in biochemistry, physiology, medicine and agriculture) occupied considerably more of the time of the biological sessions. The First International Congress of Radiobiology had been held only one month earlier in Burlington, Vermont, U.S.A., and undoubtedly this Congress, which had been organized long before the Second Geneva Conference was announced, stole much of the radiobiological thunder. Some material was presented in essentially similar form to both meetings and might thus have reached a wider audience : but if the United Nations is to organize a third conference of this sort, a better integration with meetings of international societies is desirable.

It is convenient to think of the biological effects of radiation as falling into two main classes : somatic and genetic. No startlingly new concepts were expounded in the former, but in the relatively small field of genetics one saw the unfolding of a 'new look'. It has long been axiomatic to consider the induction of true point mutations of genes as strictly linearly proportional to the dose of the inducing agentionizing radiation-irrespective of the dose-rate of the radiation. W. L. and L. B. Russell $(P / 897)$ reported results of their many years work on the induction of mutation at seven specific loci in the mouse. 'The present indications are that the induction of mutations is not linear at all doses and not independent of dose-rate. Further work is necessary to improve the precision of the observations; for if confirmed, the results indicate that new assessments will be needed of the genetic hazards in a nuclear age.

The fundamentally simple but often tedious approach to the investigation of these genetic hazards is through scoring of point mutations of genes responsible for a single characteristic ; but most of the qualities which constitute 'fitness' of an individual are determined by a multiplicity of genes. The Russells have made a first approach to investigation of fitness by scoring the size of litters and the longevity of offspring sired by an irradiated parent : both show reduction compared with controls. The 'fitness' of irradiated populations has also been investigated by Bonnier et al. $(P / 169)$ and Buzzati-Traverso and Scossiroli $(P / 1391)$ in the fruit-fly, Drosophila. The former found that, when there was strong selec. tion pressure by reducing available food supplies, the adaptation to the effects of irradiation with each generation was quicker : it appeared that the increasing heterozygosity was balancing the induced deleterious effects. The Italian workers also found this increased adaptability of the irradiated population to unfavourable environments. The irradiation induced a variability which permitted a more rapid attainment of conditions of equilibrium. There is no conflict here with the accepted law that the induced mutations are mainly detrimental in themselves, but evidence continues to accrue that the rate of evolution is enhanced even with low doses of radiation (Burdick and Mukai, $P / 895$ ).

Whereas man is certainly the ultimate object of study and the mouse is at present the nearest but Drosophila the usual model, invaluable information can be obtained even from bacteria, especially in the elucidation of chemical processes. Haas and Doudney $(P / 896)$ noted that mutation requires cellular metabolism and that starving the irradiated cell of nutrients diminishes the probability of mutation. Aminoacids and energy-sources are pre-requisites.

Of general interest to geneticists and cytogeneticists was the review by Dubinin $(P / 2074)$, presented by Prokofeva-Belgovskaya, indicating rapidly increasing activity by Soviet scientists in the fields which are generally regarded as orthodox.

When one turns to consider the somatic effects on the individual, it is found that interest is again directed to the prediction of hazard. Therefore, the relationship of dose to effect is one of the most important functions to determine. When there is but a single exposure to radiation, the objective is readily attained experimentally. For most effects the plot of effect versus dose is sigmoid; occasionally a linear relationship like the classical response for genemutation is obtained. In the latter case there is a tendency to invoke somatic mutation. There has recently, and perhaps especially in the recent report of the United Nations Scientific Committee on the Effects of Atomic Radiation, been a tendency to give more weight to linear relationships than the evidence justifies, for the confirmed examples are very few. Bond et al. $(P / 885)$ now report a further example; mammary tumours (adeno-fibroma and carcinoma, and fibro-adenoma and sarcoma) increased in SpragueDawley rats proportionately to the dose they had received. However, this linearity was obtained only by not scoring beyond ten months. There is a suggestion that the radiation was merely accelerating a natural event. Certainly somatic mutation alone could not be invoked to account for the result, since the manifestation of the tumour was shown to be dependent on humoral influences.

A linear relationship precludes a so-called threshold of dose below which no biological effects can be detected. Without attempting strictly to define the effect-dose relationship, Soviet scientists (Lebedinsky et al., $P / 2068$ ) announced the recording of the effects of very small doses of radiation. By electroencephalography, abnormalities in bio-electrical activity of the cerebral cortex have been seen with doses of the order of $1 \mathrm{r}$. A similarly sensitive system is claimed for the electroretinogram of the isolated frog's eye.

When one comes to the more difficult problems of interpreting the effects of fractionated doses or of continuous irradiation, the effect-dose relationship may be more complex. Leukæmia, a malignant disease much in the public eye at the moment and not infrequently induced by radiation, has been considered as due to somatic mutation. In mice, however, the results obtained by Mole $(P / 96)$ in two strains of mice are difficult to fit with this hypothesis, certainly in its simplest form. For 
equal accumulated doses the incidence of leukæmia within ten months was anything between 6 and 30 per cent : dose-rate or radiation-free time certainly was a relevant factor. Ford and Mole $(P / 98)$ also showed elegant pictures of the chromosomal changes found in these leukæmic cells.

Malignant disease apart, a general non-specific shortening of life-span has been attributed to the effects of radiation. It could be postulated that radiation, either as a large single dose or a multiplicity of smaller doses, could act as a non-specific stress to give this result. Curtis and Gebhard $(P / 912)$ found differences in the patterns of mortality of irradiated and non-specifically stressed mice : in their hands the latter did not cause shortening of life. Following single large doses of radiation to the whole body, the earlier deaths are considered by Maisin et al. (P/109) to be the sequelæ of irradiation of the lung, the intermediate deaths to irradiation of the head and the latest to irradiation of the abdomen. Lindop and Rotblat $(P / 292)$ also reported their preliminary results in a very large experiment designed to elucidate some of the problems of ageing. Some of the observed effects were startling, which suggests that confirmation with standard strains of mice is highly desirable.

Malignant tumours and shortening of life result from the administration of radioactive materials giving substantial radiation-doses, the pathological changes being related to the chemical characteristics of the material given and its sites of concentration. The report of Burykina et al. $(P / 2077)$ presented by Krayevsky gives details of the late effects produced by administration of fission products to experimental animals. The title of their communication suggests that the doses were small, which is true in terms of mass, but the tissue-doses were measured in kilorads. Lamerton $(P / 61)$ reviewed the problems associated with the setting of permissible doses of radioactive materials. He believes that the estimation of the radiation-dose with all its present shortcomings provides the most satisfactory criterion. $\mathrm{He}$ argues that for the induction of osteo-sarcoma there is almost certainly a threshold which must be many hundreds of rads. Much of the argument is based on the one comprehensive experiment so far carried out on the long-term toxicological effects of radioactive materials; some aspects of this work in Chicago relating to strontium-90 were given by Finkel et al. (P/911).

Whereas radiobiologists are rightly most occupied at the present time with small doses of radiation and delayed effects, there is still considerable interest in the large single dose such as might be received in an accident, or which might have to be incurred by a few to avoid harm to many. Studies have been aimed at conditioning the body to resist radiation-doses normally lethal. This has not led to any practical measure which could safely be applied, but it has led to a better understanding of the effects of radiation on the intact mammal. Hollaender et al. $(P / 898)$ summarized their latest advances in this field of chemical protection. They favour the view that the main function of these agents is neutralization of free radicals formed by ionizing radiation. In the case of other active agents, biological amines, van der Meer et al. $(P / 556)$ argue convincingly in favour of tissue hypoxia. The role of oxygen in affecting the response of cells and tissues to ionizing radiations has been widely studied and Gray et al. (P/293) reviewed some of their recent work.

In addition to exploring the possibilities of protection against lethal doses, therapy of the animal lethally irradiated has been actively investigated. Hollaender et al. $(P / 898)$ reported their results of administering blood-forming cells which recolonize the marrow spaces of the irradiated animal. This grafting of homologous tissue may lead to undesirable late reactions. Barnes et al. $(P / 97)$, by cytological methods, have noted in some of these homologous chimæras a reversion of the marrow cells back to host type; apparently the cell clones with the greatest physiological competence, be they of normal donor or radiation-damaged host, ultimately predominate. In both laboratories these therapeutic exercises have involved administration of living cells to the irradiated mouse; but cell-free chemical agents are still being tried. Soska et al. $(P / 2121)$ claim reduced mortality from irradiation following administration of deoxy nucleotides (but not following deoxynucleosides). Similarly, Panjevac et al. $(P / 490)$ found improved survival from the administration of deoxyribonucleic acid or ribonucleic acid from homologous spleen or liver, but not from testis.

Experimental work on the intact mammal has been chiefly the theme of this review. This does not mean that radiobiologists are now getting away from the simple systems - the root of the broad bean, the yeast cell and the bacterium. Many of the papers presented were concerned with the basic lesion in these test objects. Equally there were a number of papers primarily chemical, particularly on the chemistry of the nucleic acids; and a paper from the U.S.S.R. on effects of radiation on bio-lipids (Tarusov P/2248) indicates the continued interest in that country in the effects of radiation in the nervous system.

The collected papers will, of course, be published by the United Nations Organisation as the Proceedings of the Second Conference on the Peaceful Uses of Atomic Energy.

J. F. Loutit

\section{PHYSICS OF QUANTA AND PARITY}

T HE opening session of Section A (Physics) of the British Association meeting in Glasgow on August 28 was devoted to papers presented by members of the Department of Natural Philosophy in the University of Glasgow. The research work of this Department is mainly concerned with studies of photomeson production and photodisintegration, utilizing the high-energy quanta which are provided by the departmental $340-\mathrm{MeV}$. synchrotron. Three of the papers presented at this session were concerned with experimental investigations of this type; the fourth, by Prof. J. C. Gunn, professor of theoretical physics at Glasgow, discussed the important discovery during the past year of the non-conservation of parity.

In the opening address, Prof. P. I. Dee traced briefly the development of the concept of quanta from the original suggestion made by Planck, in 1901, 